Check for updates

Cite this: RSC Adv., 2017, 7, 25725

Received 25th February 2017 Accepted 21st April 2017

DOI: 10.1039/c7ra02354e

rsc.li/rsc-advances

\section{Hexagonal AgBr crystal plates for efficient photocatalysis through two methods of degradation: methyl orange oxidation and $\mathrm{Cr}^{\mathrm{VI}}$ reduction $\uparrow$}

\begin{abstract}
Hui Zheng, ${ }^{\text {ab }}$ Peng Li, ${ }^{b}$ Lisheng Gao ${ }^{b}$ and Guangming Li (DD *a
Semiconductor-based photocatalysts have been proposed owing to their potential applications in contaminated water remediation, where a silver halide exhibits more attractive performance. Herein, a hexagonal $\mathrm{AgBr}$ crystal plate has been synthesized through a facile solvothermal technique, which demonstrated high efficiency for wastewater management through two degradation pathways in the oxidation of methyl orange (MO) and the photo-reduction of hexavalent chromium $\left(\mathrm{Cr}^{\mathrm{VI}}\right)$. In order to concretely evaluate the photocatalytic performance, reaction rate constants $(\kappa)$ of $\mathrm{MO}$ and $\mathrm{Cr}^{\mathrm{Vl}}$ were calculated to be $0.43 \mathrm{~min}^{-1}$ (>400 nm), $0.73 \mathrm{~min}^{-1}$ (AM 1.5) and $0.01 \mathrm{~min}^{-1}$ (>400 nm), $0.05 \mathrm{~min}^{-1}$ (AM 1.5), which were calculated to be 2.9 and 7.7 times that of the irregular $\mathrm{AgBr}$, respectively. Furthermore, the corresponding mechanisms were also put forward to deeply understand each photocatalytic process.
\end{abstract}

\section{Introduction}

As an indispensable natural requirement for living, the conservation of water as a resource particularly groundwater should be given enough attention. However, water contamination since the industrial revolution, caused by the uncontrolled release of effluents, has attracted overwhelming public attention. The presence of refractory organic azo dyes (such as methyl orange $\mathrm{MO}$ ) and carcinogenic hexavalent chromium $\left(\mathrm{Cr}^{\mathrm{VI}}\right)$ pollution incite a serious threat to living beings. For disposal strategy, considerable applicable technologies such as solvent extraction, precipitation and photocatalytic degradation have received significant progress. ${ }^{1,2}$ Among them, semiconductor-based photocatalysis, taking clean solar energy as consuming source, have been proposed owning to its two potential degradation channels including oxidation and reduction. When light irradiates on a semiconductor, electrons absorbing sufficient energy will transfer into a conduction band (CB) and holes will be left on the valence band (VB)., ${ }^{3,4}$ Possessing suitable energy potential, electrons on $\mathrm{CB}$ will equip reduction ability while holes on VB can get oxidation performance. Holding this ascendancy, a semiconductor

${ }^{a}$ Key Laboratory of Functional Inorganic Material Chemistry (MOE), School of Chemistry and Materials Science, Heilongjiang University, No. 74, Xuefu Road, Nangang District, Harbin, Heilongjiang 150080, P. R. China. E-mail: gmli_2000@ 163.com; Tel: $+86-451-86608458$

${ }^{b}$ Institute of Forensic Science, Ministry of Public Security, No. 17 Muxidi Nanli, Beijing 100038, China

$\dagger$ Electronic supplementary information (ESI) available. See DOI: $10.1039 / \mathrm{c} 7 \mathrm{ra02354e}$ photocatalyst is capable of processing the water resource through a principle of "green chemistry" due to the "zero emission" of electric energy.

However, preeminent photocatalysts equipped with optimized activity are still exiguous and considered as an objective worth pursuing. Therefore, to gain the maximum photocatalytic properties during the reaction process, multiple ameliorations have been applied to semiconductor architecture, such as element doping, compounding with noble metal and texture engineering. ${ }^{5-7}$ The mentioned noble metal/semiconductor composite system has recently attracted significant attentions both in photocatalysis and photovoltaic conversion. ${ }^{6,8}$ Based on the surface plasmon resonance (SPR) effect of noble metals (e.g. $\mathrm{Ag}, \mathrm{Au}$ and $\mathrm{Pd}$ ), plasmonic effects enable a predominant solar energy capture and facilitate charge carrier density strengthening, which are crucial propulsions for practical photocatalytic application. ${ }^{9,10}$ Since $\mathrm{AgBr}$ was discovered to preliminarily decompose into $\mathrm{Ag}^{0}$ and could maintain stability in the early stages and successive UV illuminations, ${ }^{11}$ various silver halides ( $\mathrm{AgX}, \mathrm{X}=\mathrm{Cl}, \mathrm{Br}$ and $\mathrm{I}$ ) began to be adopted as efficient plasmonic photocatalysts, which were able to demonstrate vigorous plasmon resonance and promote photocatalytic activity. ${ }^{12-14}$

Herein, a hexagonal $\mathrm{AgBr}$ crystal plate (AgBr-hexagonal) has been synthesized through a facile solvothermal process, which demonstrates high efficiency for photocatalytic degradation performance not only in the oxidation of MO but also in photoreduction of $\mathrm{Cr}^{\mathrm{VI}}$ under visible light, as well as simulated sunlight. The morphology and state transformation of $\mathrm{AgBr}-$ hexagonal have also been explored and illustrated during and after the photocatalytic activity characterization. In order to 
strengthen the comprehension within the two photocatalytic activities, mechanisms toward the corresponding catalytic performances of such AgBr-hexagonal have been further put forward and explored in detailed. Based on the characterization mentioned above, this study looks forward to contributing significantly in the field of photocatalytic research.

\section{Experimental}

\subsection{Chemicals}

Silver nitrate $\left(\mathrm{AgNO}_{3}\right)$, sodium bromide (NaBr), glycerol, ethylene glycol and polyvinylpyrrolidone (PVP), potassium chromate $\left(\mathrm{K}_{2} \mathrm{CrO}_{4}\right)$, diphenylcarbazide (DCP), ethylene diamine tetraacetic acid solution (EDTA) were all purchased from Sinopharm Chemical Reagent Co., Ltd (Shanghai, China) and used without further processing.

\subsection{Preparation of AgBr-hexagonal}

In the synthesizing apparatus, $2.5 \mathrm{~g}$ polyvinylpyrrolidone (PVP) and $80 \mathrm{mg}$ sodium bromide $(\mathrm{NaBr})$ were both dissolved in 24 $\mathrm{mL}$ glycerol inside a round-bottom flask under $60{ }^{\circ} \mathrm{C}$ for $30 \mathrm{~min}$. Then, $56 \mathrm{mg} \mathrm{AgNO}_{3}$ resolved in $2 \mathrm{~mL}$ ethylene glycol solution was slowly injected into the above reaction system, and the temperature was retained at $60{ }^{\circ} \mathrm{C}$ for another $30 \mathrm{~min}$. Afterwards, the reaction system was warmed to $160{ }^{\circ} \mathrm{C}$ and maintained for $80 \mathrm{~min}$, and dark red solution gradually showed up during the heating process. Finally, AgBr-hexagonal was collected through rinsing, centrifugation and freezing drying. UV-visible diffuse reflectance spectrum (DRS, using $\mathrm{BaSO}_{4}$ as internal reference sample) was measured on a dry pressed disk sample using a Shimadzu UV-36000 spectrometer. The comparative photocatalyst of AgBr-irregular was synthesized through direct mixing of $\mathrm{AgNO}_{3}\left(0.02 \mathrm{~mol} \mathrm{~L}^{-1}\right)$ and $\mathrm{NaBr}$ (0.02 $\left.\mathrm{mol} \mathrm{L}^{-1}\right)$ aqueous solution under stirring.

\subsection{Characterization}

Field emission scanning electron microscope (FESEM) images, high-angel dark-field scanning transmission electron microscopy, with corresponding element images and the energydispersive X-ray analysis (EDAX), were obtained on JEOL JEM$6700 \mathrm{~F}$ at an accelerating voltage of $15 \mathrm{kV}$. X-ray diffraction analysis (XRD) was carried out using a Bruker D8 advance X-ray diffractometer with $\mathrm{Cu} \mathrm{K} \alpha$ radiation. X-ray photoelectron spectroscopy (XPS) of samples was performed on an ESCALAB MKII $\mathrm{X}$-ray photoelectron spectrometer using monochromated $\mathrm{Al} \mathrm{K} \alpha$ X-rays.

\subsection{Photocatalytic performance evaluation of $\mathrm{AgBr}$ - hexagonal}

For the photocatalytic activity assessment of such $\mathrm{AgBr}$ hexagonal, degradation of $\mathrm{MO}, \mathrm{Cr}^{\mathrm{VI}}$ as well as reduction of $\mathrm{CO}_{2}$ was carried out under visible light (>400 $\mathrm{nm}$ ) and simulated sunlight (AM 1.5). Regarding the MO photodegradation part, $20 \mathrm{mg}$ of the AgBr samples were suspended in $20 \mathrm{~mL} \mathrm{MO}$ solution with concentrations of $10 \mathrm{mg} \mathrm{mL}^{-1}$. In order to obtain dye adsorption equilibrium on the surface of catalysts, the reaction system has been stirred for 30 min under dark condition before illumination implement. Then, during the photodegradation process, a constant volume $(800 \mu \mathrm{L})$ of fixed-time reaction solution was extracted to monitor the remaining $\mathrm{MO}$ concentration via the corresponding UV-vis absorption curve. $C$ and $C_{0}$ signified the concentrations of $\mathrm{MO}$ at real-time and $10 \mathrm{mg} \mathrm{L}^{-1}$, which were transformed from the absorption value $A$ and $A_{0}$, respectively.

Distinguished from the intrinsic orange MO, the DCP method was used to colorimetrically determine the concentration of the $\mathrm{Cr}^{\mathrm{VI}}$ solution. In a typical $\mathrm{Cr}^{\mathrm{VI}}$ photoreduction procedure, an identical sample of $20 \mathrm{mg}$ photocatalyst was suspended in $19 \mathrm{~mL}$ $\mathrm{Cr}^{\mathrm{VI}}$ solution (15 $\mathrm{mg} \mathrm{L}^{-1}$ ), which was prepared from a dilute $\mathrm{K}_{2} \mathrm{CrO}_{4}$ solution. Then, $0.5 \mathrm{~mL}$ EDTA $\left(4 \mathrm{mg} \mathrm{mL}{ }^{-1}\right)$ was injected into the suspension as the sacrificial reagent, and following this the $\mathrm{pH}$ was adjusted to a value of 2 using $\mathrm{HClO}_{4}$ (20-fold dilution). Afterwards, $0.5 \mathrm{~mL}$ of $5 \mathrm{mg} \mathrm{mL}^{-1}$ DCP in acetone, as the color developing agent, was blended into the mixture causing a red-violet solution color. The $\mathrm{Cr}^{\mathrm{VI}}$ concentration was monitored through the UV-vis absorbance recording with the characteristic peak located at $540 \mathrm{~nm}$. The concentration of $\mathrm{Cr}^{\mathrm{VI}}$ at real-time and $15 \mathrm{mg} \mathrm{L}^{-1}$, was labeled as $C$ and $C_{0}$, respectively.

\section{Results and discussion}

\subsection{Synthesis and characterization}

As a general morphology controlling medium, polyvinylpyrrolidone (PVP) has been found to conglutinate with a definite crystal face so that the growth of definite crystal face was suppressed and finally specific crystal architecture could be achieved. ${ }^{15}$ Simultaneously, due to the high viscosity of both PVP and glycerol, the diffuse coefficients of both $\mathrm{Ag}^{+}$and $\mathrm{Br}^{-}$ could be slowed down leading to a further optimized shape manipulating ability. Under such controlling conditions, a homogeneous and uniform morphology of hexagonally shaped AgBr crystal was manifested in Fig. 1a-c under various magnifications. It is displayed that the average size is about 1 $\mu \mathrm{m}$. Moreover, a typical synthesis process of AgBr-hexagonal is exhibited in Fig. 1d; $\mathrm{NaBr}$ as well as $\mathrm{AgNO}_{3}$ were dissolved in a glycol solvent containing PVP as capping agent and thickener. The crystallization nucleus of AgBr was first formed at $60^{\circ} \mathrm{C}$ and then shaped into a hexagonal crystal plate with rising reaction temperature and finally maturated at $140{ }^{\circ} \mathrm{C}$. Finally, yellow colored hexagonal shaped $\mathrm{AgBr}$ was obtained (Fig. $\mathrm{S} 1 \dagger$ ). In order to disclose the uniform and exquisite morphology of $\mathrm{AgBr}$ hexagonal, the comparative photocatalyst of AgBr-irregular (Fig. S5 $\dagger$ ) was synthesized through direct mixing of $\mathrm{AgNO}_{3}$ $\left(0.02 \mathrm{~mol} \mathrm{~L}^{-1}\right)$ and $\mathrm{NaBr}\left(0.02 \mathrm{~mol} \mathrm{~L}^{-1}\right)$ aqueous solution under stirring. In Fig. S5, $\dagger$ disorganized particles with inconsistent size represent the system of AgBr-irregular. It is well known that uniform particles with smaller sizes would possess more specific surface area for reaction compared with an irregular substance. Thus, this could be the morphological reason for AgBr-hexagonal to achieve higher active photocatalytic performance than AgBr-irregular. In Fig. 1e-g, the elemental constitution and distribution were investigated through the Energydispersive X-ray analysis (EDAX) spectra and element 


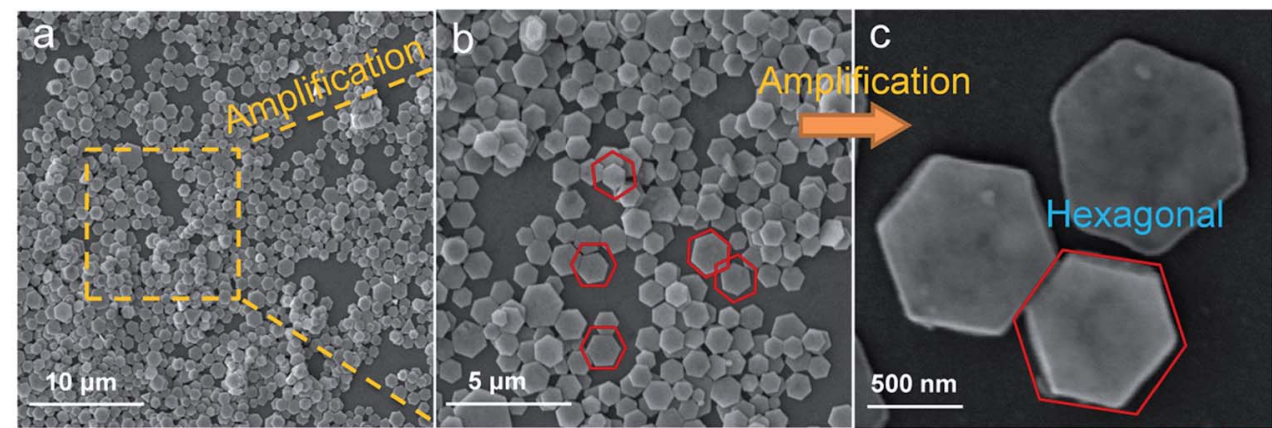

\section{d Synthesis process}

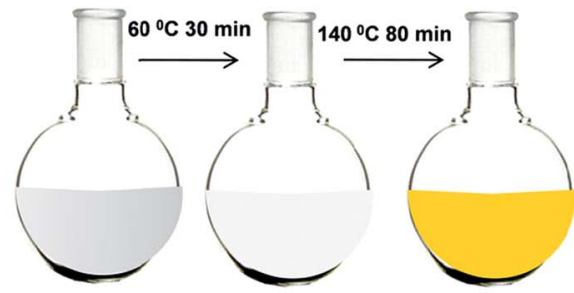

e

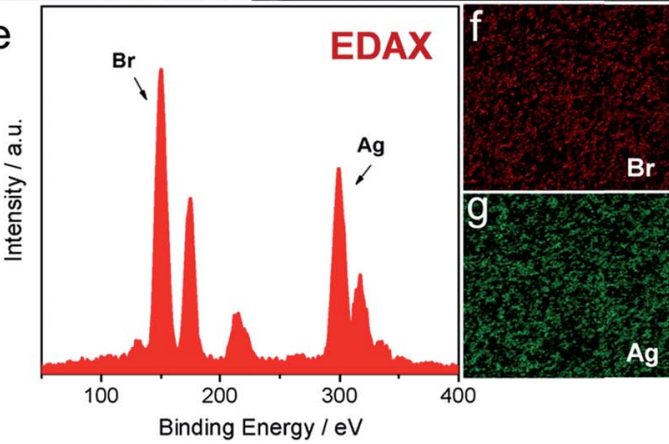

Fig. 1 (a-c) SEM images of AgBr-hexagonal crystal plate under different magnifications. (d) Typical synthesis process of AgBr-hexagonal crystal plate. $(\mathrm{e}-\mathrm{g})$ Energy-dispersive $\mathrm{X}$-ray analysis (EDAX) spectra (e) and the element mapping of $\mathrm{Br}(\mathrm{f}), \mathrm{Ag}(\mathrm{g})$ for AgBr-hexagonal crystal plate.

mapping. The two elements $\mathrm{Ag}$ and $\mathrm{Br}$ are apparently presented in the EDAX spectra in Fig. 1e, adequately conforming that the simple prepared was constituted by $\mathrm{Ag}$ and $\mathrm{Br}$ elements. In addition, the remaining elemental peaks were attributed to $\mathrm{Si}$ and $\mathrm{Au}$, which played the role of substrate and conductive enhancer, respectively. For element mapping distribution of the present AgBr-hexagonal, different colors were applied to highlight the existence of $\mathrm{Br}$ and $\mathrm{Ag}$, which further certified the sample composition, and the corresponding high-angel darkfield scanning transmission electron microscopy is demonstrated in Fig. S2. $\dagger$

The crystallographic structures of as-prepared AgBrhexagonal and normal irregular AgBr (AgBr-irregular) materials were determined via XRD analysis (Fig. 2a). Distinctive diffraction peaks $(2 \theta)$ at approximately $25.3,31.2,44.6,55.5$, 64.7 and 73.4 were manifested and assigned to (111), (200), (220), (222), (400), and (311) crystal phases of AgBr (JCPDS no. 6438). ${ }^{16}$ Moreover, no extra metallic $\mathrm{Ag}^{0}$ crystal phase was observed in the spectra indicating that solitary AgBr-hexagonal was acquired without reduction. It should be noted that no other characteristic peaks in these two crystal structures were generated, which indicated that no impurities or other crystal states were introduced during the synthesis process. As a surface chemical characterization technique, XPS was further applied to investigate the elemental composition and states of the as-prepared sample. In Fig. 2b, exhaustive elemental identification for composition analysis on the entire XPS spectrum manifested as the distinctive peaks of $\mathrm{Ag}$ and $\mathrm{Br}$ elements, dispersed on the sample surface. The element states of $\mathrm{Ag}$ and $\mathrm{Br}$ have been expressed as Ag MNN, Ag 3p, Ag 3p, Ag 3d, Ag 3d, $\mathrm{Br} 3 \mathrm{p}$ and $\mathrm{Br} 3 \mathrm{~d}$, respectively. In addition, $\mathrm{O} 1 \mathrm{~s}$ and $\mathrm{C} 1 \mathrm{~s}$ among the entire XPS spectrum were ascribed to the oxygen molecules absorbed on the sample surface and the PVP molecules. For separate analysis of elements $\mathrm{Br}$ in Fig. 2c, two respective peaks of $\mathrm{Br} 3 \mathrm{~d}_{3 / 2}$ and $3 \mathrm{~d}_{5 / 2}$ could be observed at 64.7 and $68.4 \mathrm{eV}^{17}$ Moreover, an apparent band shift to 68.5 and $69.5 \mathrm{eV}$ of $\mathrm{Br} 3 \mathrm{~d}_{3 / 2}$ and $3 \mathrm{~d}_{5 / 2}$ after photocatalytic reaction might be attributed to the generation of metallic $\mathrm{Ag}^{0}$ species on the surface of $\mathrm{AgBr}$ hexagonal, which could induce a density decrease of the electron cloud according to previous reports. ${ }^{18}$ In XPS spectra of Ag 3d states in Fig. 2d, two bands at ca. 367.7 and $373.8 \mathrm{eV}$ were ascribed to $\mathrm{Ag} 3 \mathrm{~d}_{5 / 2}$ and $\mathrm{Ag} 3 \mathrm{~d}_{3 / 2}$ (Fig. 2b), respectively. ${ }^{19}$ Due to the light sensitivity of $\mathrm{AgBr}$, metallic $\mathrm{Ag}^{0}$ nanoparticle was spontaneously generated and could be observed through the changes of XPS spectrum after photocatalytic reaction. In the XPS test result of $\mathrm{Ag} 3 \mathrm{~d}$ after photocatalytic reaction, four peaks located at 368.3, 369.3, 374.5 and $375.1 \mathrm{eV}$ were deconvoluted from the original two main peaks, where the peaks at 368.3 as well as $374.5 \mathrm{eV}$ represented $\mathrm{Ag} 3 \mathrm{~d}_{5 / 2}$ and $\mathrm{Ag} 3 \mathrm{~d}_{3 / 2}$ with other two peaks at 369.3 and $375.1 \mathrm{eV}$ indicating metallic $\mathrm{Ag}^{0}$ species. $^{\mathbf{1 4}}$ Moreover, a band shift about $0.6 \mathrm{eV}$ was also exhibited in $\mathrm{Ag} 3 \mathrm{~d}$ similar with $\mathrm{Br} 3 \mathrm{~d}$, which might have resulted from the charge density decrease around the $\mathrm{Ag}$ atoms owing to the generation of metallic $\mathrm{Ag}^{0}$. All the XPS binding energies mentioned above were consistent with reported literatures. ${ }^{{ }^{\mathbf{2 0}-22}}$

Light absorption range and intensity play an important role in photocatalysis, particularly for the visible light photodegradation of contaminants. The light harvesting capacities of the as-synthesized AgBr-irregular, AgBr-hexagonal with $\mathrm{AgBr}$ hexagonal after reaction were investigated through UV-vis diffuse reflectance absorption spectra, as shown in Fig. 3a. The intrinsic absorption edges of AgBr-hexagonal and AgBr- 

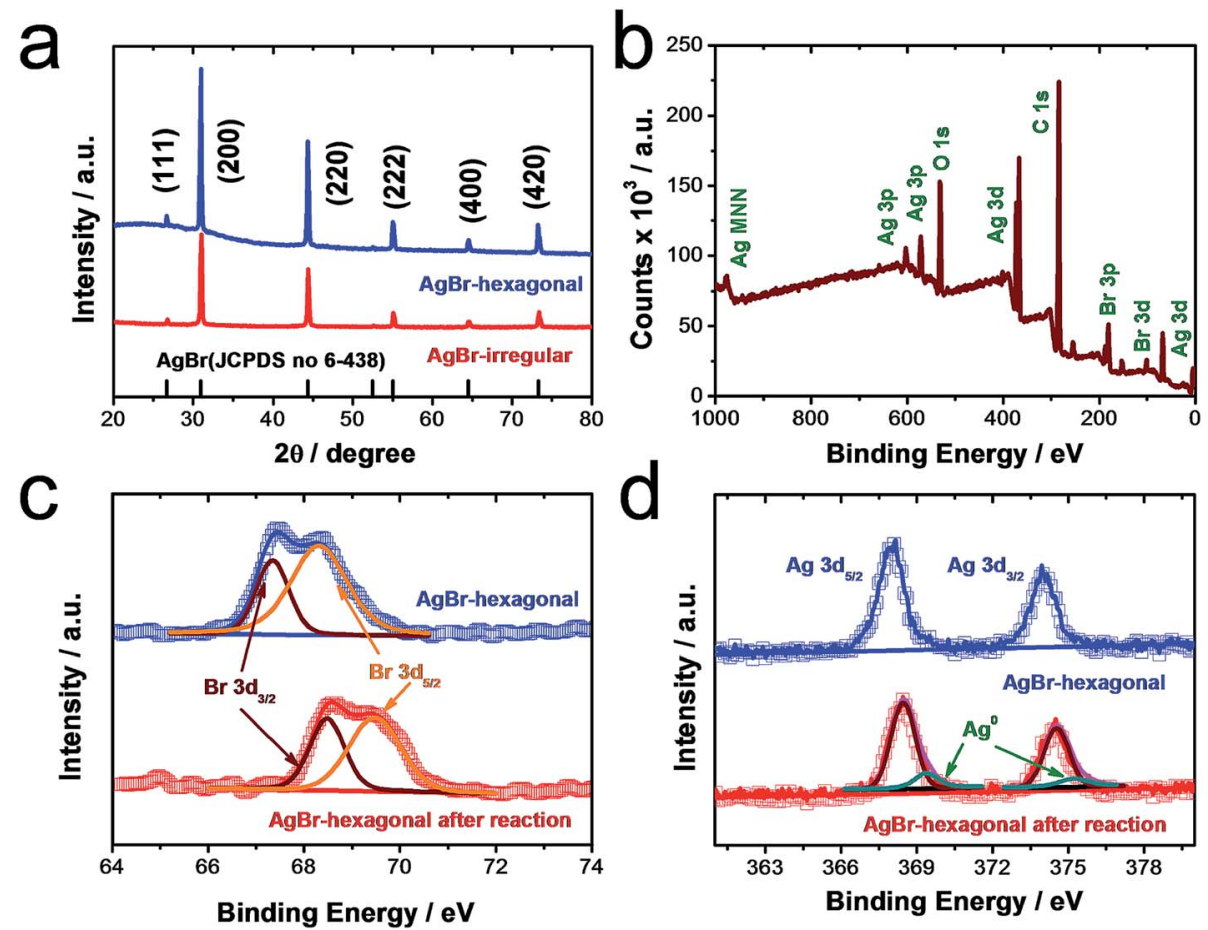

Fig. 2 (a) X-ray diffraction (XRD) analysis of both $\mathrm{AgBr}$-hexagonal crystal plate and AgBr-irregular particles. (b) Whole XPS spectrum of AgBrhexagonal crystal plate sample. (c and d) XPS spectra of $\mathrm{Br} 3 \mathrm{~d}$ (c) and $\mathrm{Ag} 3 \mathrm{~d}$ (d) from AgBr-hexagonal crystal plate before and after photocatalytic reaction.
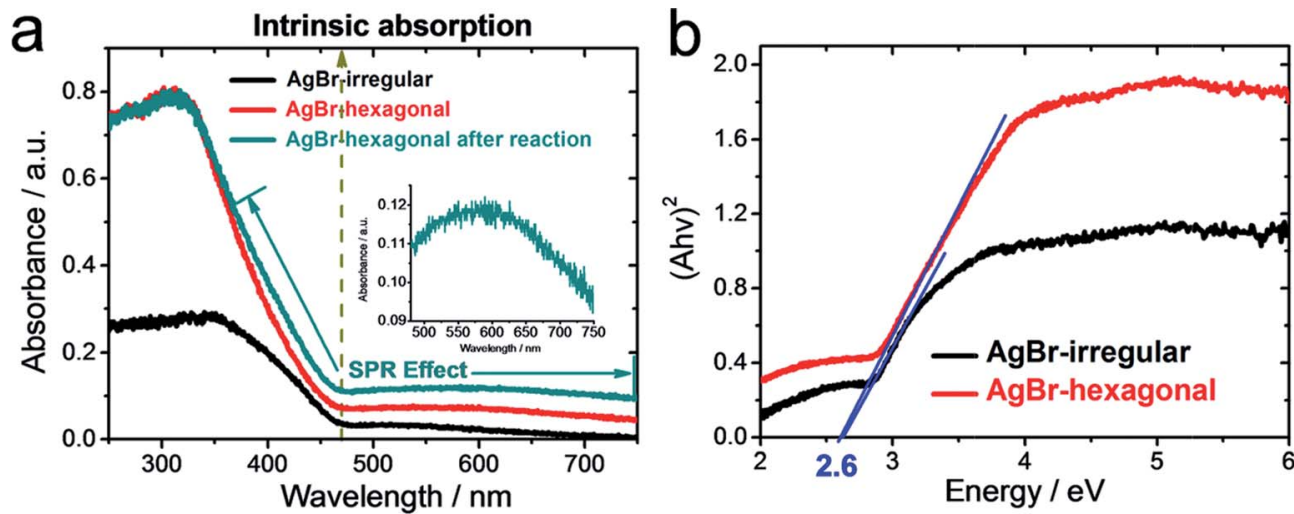

Fig. 3 (a) UV-vis diffuse reflectance absorption spectra of $\mathrm{AgBr}$-irregular, $\mathrm{AgBr}$-hexagonal and $\mathrm{AgBr}$-hexagonal after reaction, the inset is the enlargement of selected region (480-750 nm) for AgBr-hexagonal after reaction. (b) Transformed Kubelka-Munk function plot (versus the light energy) of $\mathrm{AgBr}$-irregular and $\mathrm{AgBr}$-hexagonal.

irregular were both located at $\sim 471 \mathrm{~nm}$ indicating the bandgap of $2.6 \mathrm{eV},{ }^{18}$ which could also be induced from the transformed Kubelka-Munk function plot shown (versus the light energy) in Fig. 3b. However, for the entire UV-vis range, distinctive absorption strengthening has been achieved by AgBr-hexagonal comparing with AgBr-irregular, which again illustrates the morphology advantage of the hexagonal sheet structure. Moreover, a more intensive absorption band from the $365 \mathrm{~nm}$ to $750 \mathrm{~nm}$ region was detected on the AgBr-hexagonal after one photocatalytic reaction cycle. Another such enhancement of light capture could be assigned to the localized surface plasmon resonance (LSPR) of metallic $\mathrm{Ag}^{0}$ that was inevitably generated during the photocatalytic reaction. Amplifying the selected region of AgBr-hexagonal after reaction, an absorption peak distributed, ranging from $500 \mathrm{~nm}$ to $650 \mathrm{~nm}$, might be attributed to metallic $\mathrm{Ag}^{0}{ }^{0.23,24}$ Moreover, this was another credential for the successful synthesis of unadulterated $\mathrm{AgBr}$.

\subsection{Evaluation of photocatalytic activity and corresponding mechanism}

In the wastewater treatment aspect, the photooxidation and photoreduction properties of AgBr-hexagonal crystal have been 
evaluated in the oxidative degradation of $\mathrm{MO}$ and reduction of $\mathrm{Cr}^{\mathrm{VI}}$ under visible light $(>400 \mathrm{~nm})$ irradiation and simulated solar energy (AM 1.5), respectively. The real-time concentration $\left(C_{t} / C_{0}\right)$ of the degradation object has been measured through the normalized absorption value $\left(A / A_{0}\right)$ at the given time interval. Before the photocatalytic evaluation, $30 \mathrm{~min}$ dark stirring was acquired to achieve the adsorption equilibrium, and the result is marked among the degradation curve at $0 \mathrm{~min}$ in Fig. 4a and e. As shown in Fig. 1a-c, an applanate hexagonal configuration was obtained by AgBr-hexagonal, which to a great extent promoted the molecule's adsorption ability, and this result has been shown in Fig. 4a and e. As is well known, only in the tight adjacent condition between degradation agent and photocatalyst crystal, efficient dispose effect could be gained. Thus, based on this principle, higher performance could be anticipated from AgBr-hexagonal. As intuitively revealed in Fig. $4 \mathrm{a}$ and e, comparing to the AgBr-irregular sample, there is much more predominant photocatalytic activity in MO degradation or $\mathrm{Cr}^{\mathrm{VI}}$ reduction under visible light $(>400 \mathrm{~nm})$. A pseudo-first-order reaction modus was ascribed to the photocatalytic degradation process; thus the corresponding reaction rate constant $(\kappa)$ was calculated through a linear fitting during the entire degradation process. From Fig. $4 c, d, g$ and h, MO oxidation and $\mathrm{Cr}^{\mathrm{VI}}$ reduction rate constants were estimated to be $0.43 \mathrm{~min}^{-1}$ (>400 nm), $0.73 \mathrm{~min}^{-1}$ (AM 1.5) and, $0.01 \mathrm{~min}^{-1}$ (>400 nm), $0.05 \mathrm{~min}^{-1}$ (AM 1.5), whose activity outdistanced AgBr-irregular $2.9\left(\kappa=0.15 \mathrm{~min}^{-1},>400 \mathrm{~nm} \mathrm{MO}\right)$ and 7.7 times $\left(\kappa=0.0013 \mathrm{~min}^{-1},>400 \mathrm{~nm} \mathrm{Cr}(\mathrm{vI})\right)$, respectively. As heterogeneous photocatalysis universally develops on the semiconductor surface, the reconstructed atom arrangement and electrons states on specific configuration surface should be the primary reason for catalytic promotion. ${ }^{25-27}$ Furthermore, irradiation source and intensity impact on the photocatalytic performance have also been checked between visible light and
AM 1.5. As manifested in Fig. 4a and e, distinctive photodegradation promotions were achieved using AM 1.5 as the light source in contrast with visible light, which were in accordance with photocatalytic degradation activity proportional to illumination intensity. ${ }^{8,28}$ The exhaustive UV-vis absorbance changes during the $\mathrm{MO}$ and $\mathrm{Cr}^{\mathrm{VI}}$ degradation under visible light and $\mathrm{AM}$ 1.5 irradiation were monitored, and the results are presented in Fig. S3a, b and S4a, b. $\dagger$ Apart from the degradation reactivity, another indispensable evolution index for the photocatalyst was regarded as the stability that could be reflected by means of the recycling duration. Fig. $4 \mathrm{~b}$ has listed five successive degradation cycles in oxidizing MO without palpable efficiency attenuation, which was essentially as result of the long photocatalytic stability and endurance. It was noteworthy that an appealing capability enhancement could be captured in the second cycle in contradistinction with the first curve. The interpretation could be stimulated from the generation of metallic $\mathrm{Ag}^{0}$ on the surface of AgBr-hexagonal, which could bring about the plasmonic effect, so that more robust photocatalytic efficiency could be achieved.$^{18}$ However, no apparent diversions were inspected from the five consecutive recycle processes of $\mathrm{Cr}^{\mathrm{VI}}$ reduction, as revealed in Fig. 4f. More time consumption could interpret this divergent tendency that metallic $\mathrm{Ag}^{\mathrm{O}}$ nanoparticles have completely emerged and played their role in the first circulation. Thus, no distinctive discrimination could be detected between the first and second degradation cycles. To further determine the specific surface area of AgBr-hexagonal and $\mathrm{AgBr}$ irregular, $\mathrm{N}_{2}$ adsorption-desorption isothermal analysis and pore size distribution were performed in Fig. S6. $\dagger$ Evidently, the adsorption-desorption process of AgBr-hexagonal (Fig. S6a $\dagger$ ) was irreversible, manifesting itself in the presence of a pronounced hysteresis loop. Due to the uniform and specific hexagonal sheet structure, a dense accumulation between the AgBr-hexagonal nanoparticles would be formed with stacking
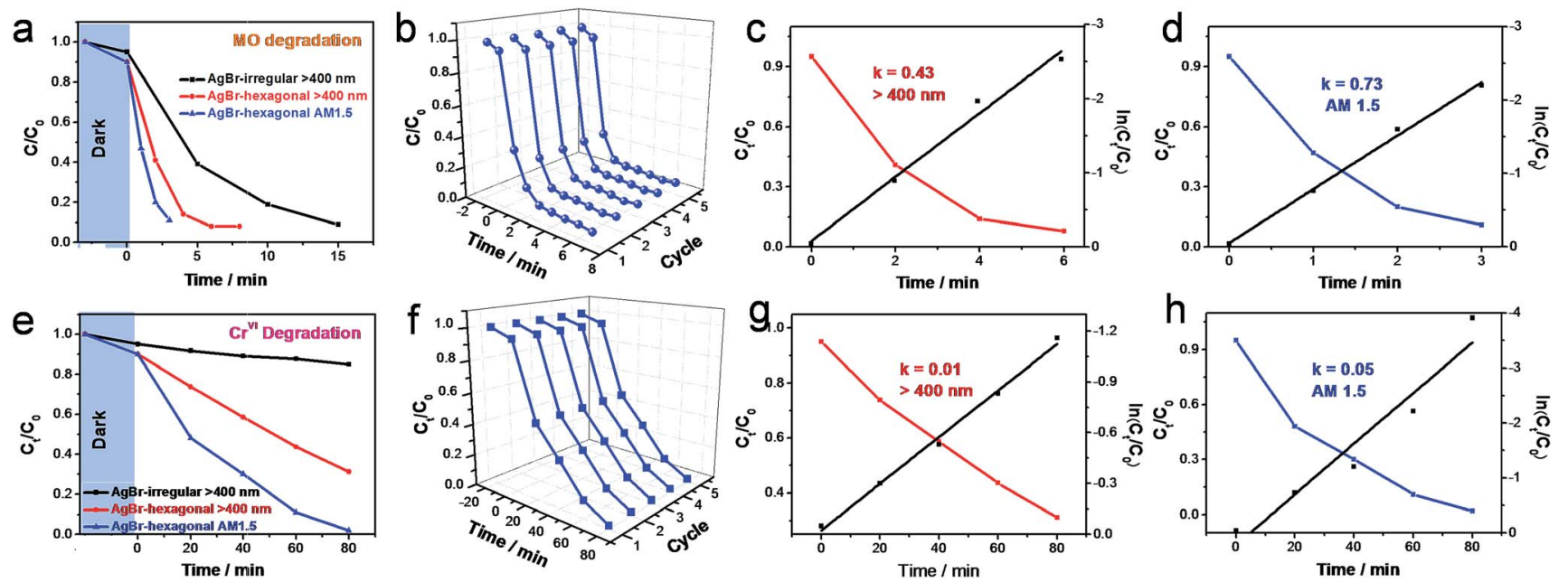

Fig. 4 (a) Photocatalytic oxidation of $\mathrm{MO}$ by AgBr-hexagonal and $\mathrm{AgBr}$-irregular under visible light (>400 nm) and simulated sunlight irradiation (AM 1.5). (b) The corresponding five successive photodegradation dynamic curves of $\mathrm{MO}$. (c and d) Corresponding reaction rate constant ( $\kappa$ ) of $\mathrm{MO}$ degradation by $\mathrm{AgBr}$-hexagonal under visible light $(>400 \mathrm{~nm})(\mathrm{c})$ and simulated sunlight (AM 1.5) (d). (e) Photocatalytic reductive curves of $\mathrm{Cr}^{\mathrm{IV}}$ over AgBr-hexagonal and $\mathrm{AgBr}$-irregular under visible light and $\mathrm{AM}$ 1.5. (f) Five successive photo-reductive dynamic curves of $\mathrm{Cr} \mathrm{r}^{\mathrm{IV}}$ by $\mathrm{AgBr}$ hexagonal crystal plate. ( $\mathrm{g}$ and $\mathrm{h}$ ) Corresponding reaction rate constant $(\kappa)$ of $\mathrm{Cr}^{\mathrm{IV}}$ degradation by $\mathrm{AgBr}$-hexagonal under visible light ( $>400 \mathrm{~nm}$ ) (g) and simulated sunlight (AM 1.5) (h). 
holes in various sizes. Shown as the pore size distribution in Fig. S6a, $\dagger$ mesopores in $2.15 \mathrm{~nm}, 2.71 \mathrm{~nm}, 3.45 \mathrm{~nm}, 4.86 \mathrm{~nm}$, $6.62 \mathrm{~nm}$ and $17.55 \mathrm{~nm}$ could be constructed by dense accumulation of AgBr-hexagonal. In addition, just a faint hysteresis loop was observed in the adsorption-desorption curve of $\mathrm{AgBr}$ irregular, which possessed $2.72 \mathrm{~nm}, 3.99 \mathrm{~nm}$ and $7.85 \mathrm{~nm}$ mesoporous. The remarkable hysteresis loop of AgBr-hexagonal comparing with AgBr-irregular might also be attributed to its plate-like morphology, which was more inclined to absorb $\mathrm{N}_{2}$ molecules. Through the calculation of the Brunauer-EmmettTeller (BET), the specific surface areas of AgBr-hexagonal and AgBr-irregular were $4.0 \mathrm{~m}^{2} \mathrm{~g}^{-1}$ and $0.3 \mathrm{~m}^{2} \mathrm{~g}^{-1}$, respectively; this was thirteen times that of the specific surface areas when $\mathrm{AgBr}$ irregular was the main explanation for the stronger dye adsorption properties of AgBr-hexagonal. Afterwards, higher photocatalytic performance would be achieved by $\mathrm{AgBr}$ hexagonal influenced by its surface dye adsorption properties.

Following this, the post-reaction AgBr-hexagonal was characterized through SEM and XRD to investigate its morphology and to constitute variations for a better understanding of the photocatalytic reaction course. As shown in Fig. $5 \mathrm{a}$, metallic $\mathrm{Ag}^{0}$ nanoparticles become evident on the exterior surface of $\mathrm{AgBr}$ hexagonal, whose appearance was deemed as an affirmative result when the halide silver was subjected to light irradiation. Moreover, a fresh peak at $38.3^{\circ}$ emerged on the XRD pattern (Fig. 5b) of the reacted AgBr-hexagonal corresponding to the (111) crystal plane of metallic $\mathrm{Ag}^{0}$ (JCPDS file: 65-2871).

In order to comprehensively understand the photocatalytic activity towards $\mathrm{MO}$ and $\mathrm{Cr}^{\mathrm{VI}}$ degradation, the relevant reaction mechanism was provided in Fig. 5c. As mentioned above, the metallic $\mathrm{Ag}^{0}$ was inevitable and began to be generated once the AgBr-hexagonal became exposed to the illumination.
Accordingly, the $\mathrm{Ag}^{0}$ modified AgBr-hexagonal was selected as the sample pattern to illustrate the mechanism during the photocatalytic activity. Once the AgBr-hexagonal semiconductor was irradiated by light, adequate energy could be received by electrons to afford the translation from the valence band (VB) to the conduction band ( $\mathrm{CB}$ ), which gave rise to the electron-hole pairs. ${ }^{29}$ Unrestrained electrons were likely to migrate to the crystal surface and combine with the $\mathrm{Ag}^{+}$resulting in metallic $\mathrm{Ag}^{0}$, whose plasmonic effect played a vital role in the photocatalytic procedure. Through the previous study in summary, two distinguishing features were embodied by the plasmonic photocatalyst. First was space charge region that could be naturally formed when metallic $\mathrm{Ag}^{\mathrm{0}}$ nanoparticle attached to the AgBr-hexagonal semiconductor with an electric field direction from AgBr-hexagonal to metallic $\mathrm{Ag}^{0}{ }^{30}$ The metallic $\mathrm{Ag}^{0}$ nanoparticles were created through the recombination of lattice $\mathrm{Ag}^{+}$with movable electrons, which resulted when the semiconductor was under illumination and such process would promote the growth of metallic $\mathrm{Ag}^{0}$ nanoparticles. However, the emergence of the internal electric field blocked the electrons migration from AgBr-hexagonal semiconductor to metallic $\mathrm{Ag}^{\mathrm{O}}$ nanoparticles, which hence impeded the $\mathrm{Ag}^{0}$ grain growth and AgBr-hexagonal crystal disintegration. Moreover, this could also be regarded as a reasonable interpretation for the positive photocatalytic repeatability of AgBr-hexagonal crystal. Second was the surface plasmon resonance (SPR) effect, possessed by the metallic $\mathrm{Ag}^{0}$ nanoparticles, that was capable of stimulating electrons under light irradiation and transfer them to the $\mathrm{CB}$ of AgBr-hexagonal semiconductor surface. ${ }^{31}$ Thus, incremental electrons were capable of participating in the photocatalytic activity and promoting its reaction performance, whether it is in wastewater treatment or in hydrocarbon fabrication. During the

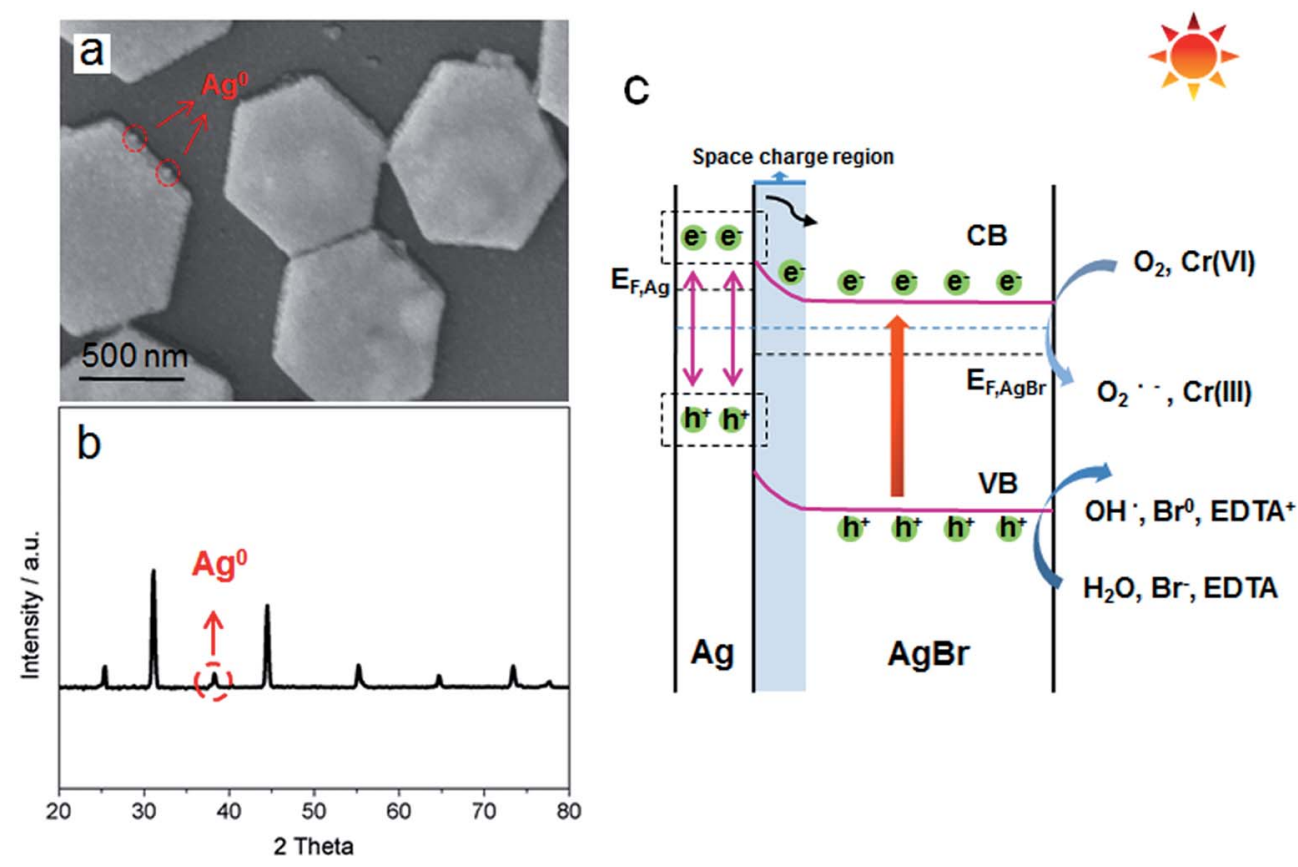

Fig. 5 (a) SEM image of AgBr-hexagonal after the photodegradation. (b) XRD pattern of the post-reaction AgBr-hexagonal. (c) Schematic diagram illustrating the speculated photocatalytic mechanism of $\mathrm{AgBr}$-hexagonal. 
organic water pollutant remediation process, the radicals have been considered as the major force in the degradation of the dye molecules, based on previous work. In the entire degradation process, radicals of $\mathrm{O}_{2}{ }^{-}-$and $\mathrm{OH}^{*}$ would be generated by $\mathrm{CB}$ electrons reacting with $\mathrm{O}_{2}$ molecules, while remaining holes combined with $\mathrm{H}_{2} \mathrm{O} .^{18,24,32,33}$ Furthermore, a few $\mathrm{Br}^{-}$ions from the AgBr-hexagonal could be oxidized into $\mathrm{Br}^{0}$ and engaged in the degradation process. ${ }^{31,33}$ For the migration of $\mathrm{Cr}^{\mathrm{VI}}$ ions from the water, enriched photo-excited electrons were equipped with the adequate energy and reduced the carcinogenic $\mathrm{Cr}^{\mathrm{VI}}$ into a benign form $\left(\mathrm{Cr}^{\mathrm{III}}\right)$, while the coordinate holes in the $\mathrm{VB}$ were trapped by EDTA as scavengers. ${ }^{18}$

\section{Conclusion}

In summary, a high performance photocatalyst AgBr-hexagonal crystal plate has been synthesized. Its activity was evaluated in two photocatalyst patterns, including MO oxidative degradation and $\mathrm{Cr}^{\mathrm{VI}}$ reductive disposing. Due to the specific hexagonal morphology, a higher adsorption behavior was achieved by AgBr-hexagonal compared with AgBr-irregular, which was considered as the main initiation factor of prominent photocatalytic activity. Besides, the degradation rate constants $(\kappa)$ of $\mathrm{MO}$ and $\mathrm{Cr}^{\mathrm{VI}}$ by AgBr-hexagonal were calculated to be 0.43 $\min ^{-1}$ (>400 nm), $0.73 \mathrm{~min}^{-1}$ (AM 1.5) and $0.01 \mathrm{~min}^{-1}(>400$ $\mathrm{nm}$ ), $0.05 \mathrm{~min}^{-1}$ (AM 1.5), which were calculated to be 2.9 and 7.7 times than for the irregular $\mathrm{AgBr}$, respectively. And corresponding mechanisms were put forward for the purpose of deeply understanding each photocatalytic process.

\section{References}

1 A. Shah, S. Shahzad, A. Munir, M. N. Nadagouda, G. S. Khan, D. F. Shams, D. D. Dionysiou and U. A. Rana, Chem. Rev., 2016, 116, 6042-6074.

2 J. Dong, J. Han, Y. Liu, A. Nakajima, S. Matsushita, S. Wei and W. Gao, ACS Appl. Mater. Interfaces, 2014, 6, 1385-1388.

3 G. Liu, J. Pan, L. Yin, J. T. S. Irvine, F. Li, J. Tan, P. Wormald and H.-M. Cheng, Adv. Funct. Mater., 2012, 22, 3233-3238.

4 S. N. Habisreutinger, L. Schmidt-Mende and J. K. Stolarczyk, Angew. Chem., Int. Ed., 2013, 52, 7372-7408.

5 G. Liu, L.-C. Yin, J. Wang, P. Niu, C. Zhen, Y. Xie and H.-M. Cheng, Energy Environ. Sci., 2012, 5, 9603-9610.

6 T. Hirakawa and P. V. Kamat, J. Am. Chem. Soc., 2005, 127, 3928-3934.

7 X. Qiu, Y. Zhao and C. Burda, Adv. Mater., 2007, 19, 3995-3999. 8 S. Bai, X. Li, Q. Kong, R. Long, C. Wang, J. Jiang and Y. Xiong, Adv. Mater., 2015, 27, 3444-3452.

9 J. He, I. Ichinose, T. Kunitake, A. Nakao, Y. Shiraishi and N. Toshima, J. Am. Chem. Soc., 2003, 125, 11034-11040.

10 R. Su, R. Tiruvalam, Q. He, N. Dimitratos, L. Kesavan, C. Hammond, J. A. Lopez-Sanchez, R. Bechstein,
C. J. Kiely, G. J. Hutchings and F. Besenbacher, ACS Nano, 2012, 6, 6284-6292.

11 N. Kakuta, N. Goto, H. Ohkita and T. Mizushima, J. Phys. Chem. B, 1999, 103, 5917-5919.

12 C. An, J. Wang, C. Qin, W. Jiang, S. Wang, Y. Li and Q. Zhang, J. Mater. Chem., 2012, 22, 13153-13158.

13 X. Wang, J. Yang, S. Ma, D. Zhao, J. Dai and D. Zhang, Catal. Sci. Technol., 2016, 6, 243-253.

14 M. Zhu, P. Chen and M. Liu, ACS Nano, 2011, 5, 4529-4536. 15 C. Han, L. Ge, C. Chen, Y. Li, Z. Zhao, X. Xiao, Z. Li and J. Zhang, J. Mater. Chem. A, 2014, 2, 12594-12600.

16 H. Wang, J. Gao, T. Guo, R. Wang, L. Guo, Y. Liu and J. Li, Chem. Commun., 2012, 48, 275-277.

17 C. Dong, K.-L. Wu, X.-W. Wei, J. Wang, L. Liu and B.-B. Jiang, Appl. Catal., A, 2014, 488, 11-18.

18 Y. Fan, W. Ma, D. Han, S. Gan, X. Dong and L. Niu, Adv. Mater., 2015, 27, 3767-3773.

19 P. Wang, Y. Tang, Z. Dong, Z. Chen and T.-T. Lim, J. Mater. Chem. A, 2013, 1, 4718-4727.

20 C. An, J. Wang, W. Jiang, M. Zhang, X. Ming, S. Wang and Q. Zhang, Nanoscale, 2012, 4, 5646-5650.

21 Y. Hou, X. Li, Q. Zhao, X. Quan and G. Chen, J. Mater. Chem., 2011, 21, 18067-18076.

22 D. Wang, L. Guo, Y. Zhen, L. Yue, G. Xue and F. Fu, J. Mater. Chem. A, 2014, 2, 11716-11727.

23 J. Belloni, M. Treguer, H. Remita and R. De Keyzer, Nature, 1999, 402, 865-867.

24 C.-C. Shen, Q. Zhu, Z.-W. Zhao, T. Wen, X. Wang and A.-W. Xu, J. Mater. Chem. A, 2015, 3, 14661-14668.

25 Y. Tang, Z. Jiang, G. Xing, A. Li, P. D. Kanhere, Y. Zhang, T. C. Sum, S. Li, X. Chen, Z. Dong and Z. Chen, Adv. Funct. Mater., 2013, 23, 2932-2940.

26 Y. G. Guo, J. S. Lee and J. Maier, Adv. Mater., 2005, 17, 28152819.

27 Y. Zhao, L. Kuai and B. Geng, Catal. Sci. Technol., 2012, 2, 1269-1274.

28 W. Yao, B. Zhang, C. Huang, C. Ma, X. Song and Q. Xu, J. Mater. Chem., 2012, 22, 4050-4055.

29 N. Feng, Q. Wang, A. Zheng, Z. Zhang, J. Fan, S.-B. Liu, J.-P. Amoureux and F. Deng, J. Am. Chem. Soc., 2013, 135, 1607-1616.

30 P. Zhou, J. Yu and M. Jaroniec, Adv. Mater., 2014, 26, 49204935.

31 J. Gamage McEvoy, W. Cui and Z. Zhang, Appl. Catal., B, 2014, 144, 702-712.

32 D. Wang, Y. Duan, Q. Luo, X. Li, J. An, L. Bao and L. Shi, J. Mater. Chem., 2012, 22, 4847-4854.

33 X. Wang, Y. Tang, Z. Chen and T.-T. Lim, J. Mater. Chem., 2012, 22, 23149-23158. 